\title{
ELECTROMOTIVE FORCES OF CONCENTRATION CELLS
}

\author{
BY J. E. TREVOR
}

CONTENTS

The problem. The electromotive force of the cell. Helmholtz's equation. Integration of Helmholtz's equation. Integration with regard to the degree of electrolytic dissociation. The assumptions made. Integration assuming the dilution law of Rudolphi-van't Hoff.

\section{The problem}

For the electromotive force of a concentration cell of the type

$$
\mathrm{M}|\mathrm{MCl}, \mathrm{I}| \mathrm{HgCl} . \mathrm{Hg} . \mathrm{HgCl}|\mathrm{MCl}, \mathrm{II}| \mathrm{M},
$$

Helmholtz, in 1882 , under certain simplifying assumptions, deduced the equation

$$
\mathrm{A}_{\mathrm{I}}-\mathrm{A}_{\mathrm{II}}=q \cdot r_{2} \theta \int_{h_{\mathrm{II}}}^{h_{\mathrm{I}}} h \frac{\partial \log p_{1}}{\partial \bar{h}} \partial h .
$$

Here $q$ denotes the mass of salt dissolved on the passage of unit current in unit time, $r_{2}$ the specific gas-constant of water vapor, $\theta$ the absolute temperature, $h=\mathrm{M}_{2} / \mathrm{M}_{1}$ the mass of water per unit mass of salt in the solution, and $p_{1}(\theta, h)$ the vapor pressure of the solution.

For comparison with experiment, Helmholtz evaluated the integral in ( $\mathrm{I}$ ) by means of an empirical relation giving the vapor pressure of solutions of zinc chloride as a function of the concentration at constant temperature. The object of the present note is to integrate Helmholtz's differential equation under the assumption that the relation between vapor pressure and concentration is given by the equation of the' theory of dilute solutions,

$$
\log \frac{p_{0}}{p_{1}}=(\mathrm{I}+\alpha) \frac{n_{1}}{n_{2}}
$$


together with Ostwald's dilution law

$$
k \frac{\mathrm{I}-\alpha}{\mathrm{U}}=\left(\begin{array}{c}
\alpha \\
\overline{\mathrm{U}}
\end{array}\right)^{2}
$$

and to determine how the result is altered when Ostwald's equation is replaced by van't Hoff's modification

$$
\mathrm{K}\left(\frac{\mathrm{I}-\alpha}{\mathrm{U}}\right)^{2}=\left(\frac{\alpha}{\mathrm{U}}\right)^{3}
$$

of the form proposed by Rudolphi for the dilution law. In these equations, $p_{0}(\theta)$ denotes the vapor pressure of water, $n_{1} / n_{2}$ the number of mols of salt in the solution per mol of water, $\alpha$ the degree of electrolytic dissociation of the (binary) salt, and $U$ the volume of the solution per mol of salt.

\section{The electromotive force of the cell}

For the purpose of making apparent the assumptions involved by (I), I shall begin by deducing the general thermodynamic equation for the electromotive force of the cell. Let $\mathrm{F}\left(p, \theta, \mathrm{M}_{1}, \mathrm{M}_{2}\right)$ be the free energy of the solution composed of the masses $M_{1}, M_{2}$ of salt and water under the pressure $p$ at the temperature $\theta$. The change of the free energy of the system composed of these masses, when the separated masses at $p, \theta$ reversibly or irreversibly form the solution stable at $p, \theta$, is

$$
\Delta \mathrm{F}=\mathrm{F}-\mathrm{M}_{1} f_{1}-\mathrm{M}_{2} f_{2},
$$

where $f_{j}(p, \theta)$ is the specific free energy of the $j$-th component. Since this function $\Delta \mathrm{F}$ of the variables $p, \theta, \mathrm{M}_{1}, \mathrm{M}_{2}$ is homogeneous and of the first degree in $\mathrm{M}_{1}, \mathrm{M}_{2}$, we have

$$
\Delta \mathrm{F}=\mathrm{M}_{1} \frac{\partial \Delta \mathrm{F}}{\partial \mathrm{M}_{1}}+\mathrm{M}_{2} \frac{\partial \Delta \mathrm{F}}{\partial \mathrm{M}_{2}}
$$

whence, differentiating, with regard to either $\mathrm{M}_{1}$ or $\mathrm{M}_{2}$,

$$
\frac{\partial}{\partial h} \frac{\partial \Delta \mathrm{F}}{\partial \mathrm{M}_{1}}+h \frac{\partial}{\partial h} \frac{\partial \Delta \mathrm{F}}{\partial \mathrm{M}_{2}}=0 .
$$

This general equation may be employed to connect the electromotive force of the concentration cell with the concentrations of the two solutions. For $\partial \Delta \mathrm{F} / \partial \mathrm{M}_{1}$ may be 
expressed by means of the electromotive force of the single cell, two of which constitute the concentration cell; and $\partial \Delta \mathrm{F} / \partial \mathrm{M}_{2}$ may be expressed by means of the vapor pressure of the solution and thus by means of the concentration; wherefore integration between the two concentrations of the double cell will yield the relation desired.

Let the current in the single cell flow from the metal M to the mercury. When the current $\mathrm{J}$ flows in the time $d t$, the electromotive force $\mathrm{A}$ does the work $\mathrm{AJdt}$. The work added to the solution is -AJdt. This work may also be expressed as $\partial \Delta \mathrm{F} / \partial \mathrm{M}_{1} . J q d t$. This work being exclusively electrical work,

$$
\begin{gathered}
-\mathrm{A} J d t=\frac{\partial \Delta \mathrm{F}}{\partial \mathrm{M}_{\mathrm{L}}} \mathrm{J} q d t \\
\frac{\partial \Delta \mathrm{F}}{\partial \mathrm{M}_{1}}=-\frac{\mathrm{A}}{q} \\
\frac{\partial}{\partial h} \frac{\partial \Delta \mathrm{F}}{\partial \mathrm{M}_{1}}=-\frac{\mathrm{I}}{q} \frac{\partial \mathrm{A}}{\partial h},
\end{gathered}
$$

as was shown by Helmholtz.

On p. 399 of a paper ${ }^{1}$ on the thermodynamic theory of solutions, I have shown, equation (15a), that

$$
\frac{\partial \Delta \mathrm{F}}{\partial \mathrm{M}_{2}}=p^{2} \frac{\partial}{\partial p} \frac{\mathrm{I}}{\partial}\left\{\int_{p_{1}}^{p_{0}}\left(v_{a}-\frac{\partial \mathrm{V}}{\partial \mathrm{M}_{2}}\right) d p+\int_{p_{0}}^{p}\left(z_{2}-\frac{\partial \mathrm{V}}{\partial \mathrm{M}_{2}}\right) d p\right\},
$$

where $v_{a}(p, \theta)$ is the specific volume of saturated water vapor, $v_{2}(p, \theta)$ is the specific volume of liquid water, and $V\left(p, \theta, M_{1} M_{2}\right)$ is the volume of the solution of $\mathrm{M}_{1}$ in $\mathrm{M}_{2}$. Executing the indicated differentiation,

$$
\frac{\partial \Delta \mathrm{F}}{\partial \mathrm{M}_{2}}=p\left(v_{2}-\frac{\partial \mathrm{V}}{\partial \mathrm{M}_{2}}\right)-\int_{p_{1}}^{p_{0}}\left(v_{a}-\frac{\partial V}{\partial \mathrm{M}_{2}}\right) d p-\int_{p_{0}}^{p}\left(v_{2}-\frac{\partial V}{\partial \mathrm{M}_{2}}\right) d p ;
$$

whence

$$
\begin{aligned}
\frac{\partial}{\partial h} \frac{\partial \Delta \mathrm{F}}{\partial \mathrm{M}_{2}}=-p \frac{\partial}{\partial h} \frac{\partial \mathrm{V}}{\partial \mathrm{M}_{2}}-\frac{\partial}{\partial h} \int_{p_{1}}^{p_{0}}\left(v_{l t}-\frac{\partial \mathrm{V}}{\partial \mathrm{M}_{2}}\right) d p & \\
& -\frac{\partial}{\partial h} \int_{p_{0}}^{p}\left(v_{2}-\frac{\partial \mathrm{V}}{\partial \mathrm{M}_{2}}\right) d p .
\end{aligned}
$$

1 This Journal, 10, 392 (1906). 
Substituting (3) and (4) in the general equation (2), solving for $\partial \mathrm{A} / \partial h$, and integrating between $h_{\mathrm{II}}$ and $h_{\mathrm{s}}$, where $h_{1}>h_{\mathrm{s}}$,

(5) $\begin{aligned} \mathrm{A}_{\mathrm{I}}-\mathrm{A}_{\mathrm{II}}=-q p \int_{h_{1 \mathrm{I}}}^{h_{\mathrm{I}}} h \frac{\partial}{\partial h} \frac{\partial \mathrm{V}}{\partial \mathrm{M}_{2}} d h & -q \int_{h_{\mathrm{II}}}^{h_{\mathrm{I}}} h \frac{\partial}{\partial h} \int_{p_{1}}^{\phi_{0}}\left(v_{a}-\frac{\partial \mathrm{V}}{\partial \mathrm{M}_{2}}\right) d p . d h \\ & -q \int_{h_{\mathrm{II}}}^{h_{h_{\mathrm{I}}}} \frac{\partial}{\partial h} \int_{p_{0}}^{\phi}\left(v_{2}-\frac{\partial \mathrm{V}}{\partial \mathrm{M}_{2}}\right) d p \cdot d h .\end{aligned}$

This is the wholly general thermodynamic equation for the electromotive force of the concentration cell at $p, \theta$. When the cell functions under the pressure $p=p_{0}$, the last of the three terms of the second member disappears.

\section{Helmholtz's equation}

If we assume that $p=p_{0}$, and also that $\partial \mathrm{V} / \partial \mathrm{M}_{2}$ is independent of $h$ and is negligible in comparison with $v_{a}$, the equation (5) becomes

$$
\mathrm{A}_{\mathrm{I}}-\mathrm{A}_{\mathrm{II}}=-q \int_{h_{\mathrm{II}}}^{h_{\mathrm{I}}} h \frac{\partial}{\partial h} \int_{p_{1}}^{p_{0}} v_{a} d p . d h .
$$

If we further assume that the saturated water vapor may be regarded as an ideal gas, $i$. $e$., that $v_{a}=r_{2} \theta / p$, we find

$$
\mathrm{A}_{\mathrm{I}}-\mathrm{A}_{\mathrm{II}}=q \cdot r_{2} \theta \int_{h_{\mathrm{II}}}^{h_{\mathrm{I}}} h \frac{\partial \log p_{1}}{\partial h} d h,
$$

which is Helmholtz's equation as cited at the beginning of this article.

\section{Integration of Helmholtz's equation}

Our present problem is to effect the integration of $(5 a)$, under the assumption that the form of $p_{1}(\theta, h)$ is given by

$$
\log \frac{p_{0}}{p_{1}}=(\mathbf{1}+\alpha) \frac{n_{1}}{n_{2}}
$$

where the form of $\alpha(\theta, h)$ is to be obtained from

$$
k^{\mathrm{I}} \frac{\alpha}{\overline{\mathrm{U}}^{-\alpha}}=\left(\frac{\alpha}{\overline{\mathrm{U}}}\right)^{2} \text {. }
$$


We thus require to find the form of $\partial \log p_{1} / \partial h$ in $h$, through elimination of $\alpha$ between (6), (7), and determination of the forms of the functions $n_{1} / n_{2}$ and $\mathrm{U}$.

Solving (7) for $\alpha$,

$$
\alpha=-\frac{k \mathrm{U}}{2}+\left\{k \mathrm{U}+\left(\frac{k \mathrm{U}}{2}\right)^{2}\right\}^{\frac{1}{2}}
$$

Here, both $k$ and $U$ being positive, the positive square root must be taken to make $\alpha$ positive. Eliminating $\alpha$,

$$
-\log \frac{p_{\mathrm{O}}}{p_{1}}=-\frac{n_{1}}{n_{2}}+\frac{k \mathrm{U}}{2} \frac{n_{1}}{n_{2}}-\frac{n_{1}}{n_{2}}\left\{k \mathrm{U}+\left(\frac{k \mathrm{U}}{2}\right)^{2}\right\}^{\frac{1}{2}}
$$

It remains to express $n_{1} / n_{2}$ and $\mathrm{U}$ in terms of $h$. Writing $w_{j}$ for the molecular weight of the $j$-th component of the solution,

$$
\frac{n_{1}}{n_{2}}=\frac{\mathrm{M}_{1}}{\mathrm{M}_{2}} \frac{w_{2}}{w_{1}}=\frac{w_{2}}{w_{1}} \frac{\mathrm{I}}{h}
$$

Identifying the volume $\mathrm{V}$ of the solution with the mass of water in the solution, $\mathrm{V}=\mathrm{M}_{2}$; whence $\mathrm{U}$, the volume of the solution per mol of salt, becomes

$$
\mathrm{U}=\frac{\mathrm{V}}{\mathrm{M}_{1} / w_{1}}=\frac{w_{1} \mathrm{M}_{2}}{\mathrm{M}_{1}}=w_{1} h
$$

Eliminating $n_{1} / n_{2}$ and $\mathrm{U},(8)$ becomes

$$
\text { (II) }-\log \frac{p_{0}}{p_{1}}=-\frac{w_{2}}{w_{1}} \frac{\mathbf{I}}{h}+\frac{k w_{2}}{2}-\frac{w_{2}}{w_{1}} \frac{\mathrm{I}}{h}\left\{k w_{1} h+\left(\frac{k w_{1} h}{2}\right)^{2}\right\}^{\frac{1}{2}} \text {. }
$$

Differentiating,

$$
\frac{\partial \log p_{1}}{\partial h}=\frac{w_{2}}{w_{1}} \frac{\mathbf{I}}{h^{2}}+\frac{w_{2}}{w_{1}} \frac{k w_{1}}{2 h\left(k w_{1} h+k^{2} w_{1}^{2} / 4 \cdot h^{2}\right)^{\frac{1}{2}}}
$$

Substituting this expression in $(5 a)$, and noting that $w_{2} r_{2}$ is the molecular gas-constant $r$,

$$
\begin{gathered}
\mathrm{A}_{\mathrm{I}}-\mathrm{A}_{\mathrm{II}}=\frac{q}{w_{1}} \cdot r \theta \cdot \log \frac{h_{\mathrm{I}}}{h_{\mathrm{II}}}+\frac{k q \cdot r \theta}{2} \int_{h_{\mathrm{II}}}^{h_{\mathrm{I}}} \frac{d h}{\left(k w w_{1} h+k^{2} w_{1}^{2} / 4 \cdot h^{2}\right)^{\frac{3}{2}}} \\
\text { (13) }=\left.\frac{q}{w_{1}} \cdot r \theta \cdot \log h\left\{\mathrm{r}+\frac{k w_{1}}{2} h+\sqrt{k w_{1} h+\left(\frac{k w_{1} h}{2}\right)^{2}}\right\}\right|_{h_{\mathrm{II}}} ^{h_{\mathrm{I}}} .
\end{gathered}
$$


This result assumes a simpler form when the composition of the solution is expressed by means of $\alpha$ instead of by means of $h$. From (7) and (Io), namely

$$
k \mathrm{U}=\frac{\alpha^{2}}{\mathrm{I}-\alpha}, \quad \mathrm{U}=w_{1} h,
$$

we find $h(\alpha)$ to be

$$
\frac{\mathrm{I}}{k w_{1}} \mathrm{\alpha}-\alpha
$$

Replacing $h$ in (1 3 ) by $h(\alpha)$, we find

$$
\begin{gathered}
\mathrm{A}_{\mathrm{I}}-\mathrm{A}_{\mathrm{II}}=\left.2 \frac{q}{w_{1}} \cdot r \theta \cdot \log \frac{\alpha}{\mathrm{I}-\alpha}\right|_{\alpha_{\mathrm{II}}} ^{\alpha_{\mathrm{I}}} \\
=2 \frac{q}{w_{1}} \cdot r \theta \cdot \log \frac{\alpha_{\mathrm{I}}}{\alpha_{\mathrm{II}}} \frac{\mathrm{I}-\alpha_{\mathrm{II}}}{\mathrm{I}-\alpha_{\mathrm{I}}} .
\end{gathered}
$$

\section{Interration with regard to the degree of electrolytic dissociation}

As is indicated by the simple form of the result ( $13 a$ ), the above integration of Helmholtz's differential equation is more readily effected when $\alpha$ instead of $h$ is employed as the concentration variable. This shall now be shown. From the general thermodynamic equation

$$
\Delta \mathrm{F}=\mathrm{M}_{1} \frac{\partial \Delta \mathrm{F}}{\partial \mathrm{M}_{1}}+\mathrm{M}_{2} \frac{\partial \Delta \mathrm{F}}{\partial \mathrm{M}_{2}},
$$

by differentiation with regard to $\mathrm{M}_{2}$ we find

$$
\left(M_{1} \frac{\partial}{\partial \alpha} \frac{\partial \Delta F}{\partial M_{1}}+M_{2} \frac{\partial}{\partial \alpha} \frac{\partial \Delta F}{\partial M_{2}}\right) \frac{\partial \alpha}{\partial M_{2}}=0 .
$$

Noting that $\partial \alpha / \partial M_{2}$ does not vanish, and that

$$
\frac{\mathrm{M}_{2}}{\overline{\mathrm{M}}_{1}}==h=\frac{\mathrm{I}}{k w w_{1}} \frac{\alpha^{2}}{\mathrm{I}-\alpha},
$$

as just shown, we obtain

$$
\frac{\partial}{\partial \alpha} \frac{\partial \Delta \mathrm{F}}{\partial \bar{M}_{1}}+\frac{\mathbf{I}}{k w_{1}} \frac{\alpha^{2}}{\mathbf{I}-\alpha} \frac{\partial}{\partial \alpha} \frac{\partial \Delta \mathrm{F}}{\partial \mathrm{M}_{2}}=0 .
$$

Now, as before

$$
\frac{\partial \Delta \mathrm{F}}{\partial \mathrm{M}_{1}}=-\frac{\mathrm{A}}{q}
$$


whence

$$
\frac{\partial}{\partial \alpha} \frac{\partial \Delta \mathrm{F}}{\partial \mathrm{M}_{\mathbf{1}}}=-\frac{\mathrm{I}}{q} \frac{\partial \mathrm{A}}{\partial \alpha} .
$$

Again as before, and under the same assumptions,

$$
\begin{aligned}
\frac{\partial \Delta \overrightarrow{\mathrm{F}}}{\partial \overline{\mathrm{M}}_{2}} & =-\int_{p_{1}}^{p_{o}} v_{a}(p, \theta) d p \\
& =-\phi\left(p_{0}(\theta), \theta\right)+\phi\left(p_{1}(\theta, \alpha), \theta\right) ;
\end{aligned}
$$

whence

$$
\begin{aligned}
\frac{\partial \partial \mathrm{\partial} \boldsymbol{F}}{\partial \alpha \mathrm{M}_{2}} & =\frac{\partial \phi}{\partial p_{1}} \frac{\partial p_{1}}{\partial \alpha} \\
& =v_{a}\left(p_{1}, \theta\right) \frac{\partial p_{1}}{\partial \alpha} \\
& =r_{2} \theta \frac{\partial \log p_{1}}{\partial \alpha} .
\end{aligned}
$$

Again assuming (6), we have

$$
\begin{aligned}
\log \underset{p_{\mathrm{O}}}{p_{0}} & =(\mathrm{I}+\alpha) \frac{n_{1}}{n_{2}} \\
& =(\mathrm{I}+\alpha) \frac{w_{2}}{w_{1}} h \\
& =2 w_{2} k \frac{\mathrm{I}-\alpha^{2}}{\alpha^{2}} \\
\frac{\partial \log p_{1}}{\partial \alpha} & =\frac{2 w_{2} k}{\alpha^{3}} ;
\end{aligned}
$$

wherefore (16) becomes

$(16 a)$

$$
\frac{\partial}{\partial \alpha} \frac{\partial \Delta \mathrm{F}}{\partial \mathrm{M}_{2}}=\frac{2 k \cdot w_{2} r_{2} \cdot \theta}{\alpha^{3}} .
$$

Substituting (I5) and (I6a) in (I4),

$$
\partial \mathrm{A}=2 \frac{q}{w_{1}} r \theta \frac{\mathrm{I}}{(\mathrm{I}-\alpha) \alpha} .
$$

Integrating over the difference of the concentrations in the concentration cell,

$$
\mathrm{A}_{\mathrm{I}}-\mathrm{A}_{\mathrm{II}}=\left|2 \frac{q}{w_{1}} r \theta \log \frac{\alpha}{\mathrm{I}-\alpha}\right|_{\alpha_{\mathrm{II}}}^{a_{\mathrm{I}}}
$$

the same result $\left(\mathrm{r}_{3} a\right)$ as before. 


\section{The assumptions made}

From the above work it is clear that Helmholtz's differential equation ( $\mathbf{I}$ ) involves the assumptions:

The salt is involatile;

The cell functions under the pressure $p_{0}(\theta)$;

$\partial \mathrm{V} / \partial \mathrm{M}_{2}$ is independent of $h$, and negligible

in comparison with $v_{a}$;

The form of $v_{a}(p, \theta)$ is $r_{2} \theta \mid p$.

And it is clear that the further assumptions made in the integration, and thereby involved in the equivalent formulations ( 13$),(\mathrm{I} 3 a)$, are:

$$
\begin{aligned}
& \log \frac{p_{0}}{p_{1}}=(\mathrm{I}+\alpha) \frac{n_{1}}{n_{2}} \\
& k \frac{\mathrm{I}-\alpha}{\mathrm{U}}=\frac{\alpha^{2}}{\mathrm{U}^{2}} \\
& \mathrm{~V}=\mathrm{M}_{2} \text {. I. e. the volume of the solution in } \mathrm{cm}^{3} \text { is sensibly } \\
& \text { the same as the mass of the water in grams. }
\end{aligned}
$$

It will not be overlooked that the first of these further assimptions introduces the hypothesis that the heats of dilution of the solutions are negligibly small. The general equation (5) for the electromotive force of the concentration cell involves only the assumption of the involatility of the salt.

\section{Integration assuming the dilution law of Rudolphi-van't Hoff}

It is not without interest to determine the manner in which the foregoing result is modified when the assumption of Ostwald's dilution law is replaced by that of Rudolphi's equation as modified by van't Hoff,

$$
\mathrm{K}\left(\frac{\mathrm{r}-\alpha}{\mathrm{U} \cdot}\right)^{2}=\left(\frac{\alpha}{\mathrm{U}}\right)^{3}
$$

The formulation $\mathrm{U}=w_{1} h$ of equation (Io) converts this to

$$
h=\frac{\mathrm{I}}{w_{1} \mathrm{~K}} \frac{\alpha^{3}}{(\mathrm{I}-\alpha)^{2}}
$$


wherefore the general relation

$$
\frac{\partial}{\partial \alpha} \frac{\partial \Delta F}{\partial M_{1}}+h \frac{\partial}{\partial \alpha} \frac{\partial \Delta F}{\partial \bar{M}_{2}}=0
$$

becomes

$$
\frac{\partial}{\partial \alpha} \frac{\partial \Delta \mathrm{F}}{\partial M_{1}}+\frac{\mathrm{I}}{w_{1} \mathrm{~K}} \frac{\alpha^{3}}{(\mathrm{I}-\alpha)^{2}} \frac{\partial \Delta \mathrm{F}}{\partial \mathrm{M}_{2}}=0 .
$$

As before, equations ( 15 ), ( 16 ), we have

$$
\frac{\partial}{\partial \alpha} \frac{\partial \Delta \mathrm{F}}{\partial \mathrm{M}_{1}}=-\frac{\mathrm{I}}{q} \frac{\partial \mathrm{A}}{\partial \alpha}, \quad \frac{\partial}{\partial \alpha} \frac{\partial \Delta \mathrm{F}}{\partial \mathrm{M}_{2}}=r_{2} \theta \frac{\partial \log p_{1}}{\partial \alpha} ;
$$

wherefore

$$
\frac{\partial \mathrm{A}}{\partial \alpha}=\frac{q}{z w_{1}} \frac{r_{2} \theta}{\mathrm{K}} \frac{\alpha^{3}}{(\mathrm{I}-\alpha)^{2}} \frac{\partial \log \underline{p}_{1}}{\partial \alpha} .
$$

Now, by (6), (9), ( $\mathrm{I} 7)$,

$$
\begin{aligned}
& \log \frac{p_{0}}{p_{1}}=w_{2} \mathrm{~K} \frac{(\mathrm{I}+\alpha)(\mathrm{1}-\alpha)^{2}}{\alpha^{3}} \\
& \frac{\partial \log p_{1}}{\partial \alpha}=w_{2} \mathrm{~K} \frac{(3+\alpha)(1-\alpha)}{\alpha^{4}} ;
\end{aligned}
$$

which converts (I8) to

$$
\frac{\partial \mathrm{A}}{\partial \alpha}=\frac{q}{v v_{1}} r \theta \frac{3+\alpha}{(\mathbf{1}-\alpha) \alpha}
$$

wherefore; integrating between $\alpha_{\mathrm{II}}, \alpha_{1}$,

$$
\mathrm{A}_{\mathrm{I}}-\mathrm{A}_{\mathrm{II}}=\left.\frac{q}{w_{1}} r \theta\left(3 \log \frac{\alpha}{\mathrm{I}-\alpha}+\log \frac{\mathrm{I}}{\mathrm{I}-\alpha}\right)\right|_{\alpha_{\mathrm{II}}} ^{\alpha_{\mathrm{I}}} .
$$

This is the formulation sought. It may be written

$$
\begin{aligned}
\mathrm{A}_{\mathrm{I}}-\mathrm{A}_{\mathrm{II}} & =\left.\frac{q}{w_{1}} r \theta \log \frac{\alpha^{3}}{(\mathrm{I}-\alpha)^{4}}\right|_{\alpha_{\mathrm{II}}} ^{\alpha_{\mathrm{I}}} \\
& =\frac{q}{w_{1}} r \theta \log \frac{\alpha_{\mathrm{I}}^{\mathrm{I}}}{\alpha_{\mathrm{II}}^{8}} \frac{\left(\mathrm{I}-\frac{\left.\alpha_{\mathrm{II}}\right)^{4}}{\left(\mathrm{I}-\alpha_{\mathrm{I}}\right)^{4}} .\right.}{}
\end{aligned}
$$

Comell University, January, 1907 